\title{
Patterns of recurrence and survival in breast cancer
}

\author{
DANIELA NAVARRETE MONTALVO ${ }^{1}$, NICOLÁS GONZÁLEZ M ${ }^{1}$, MARÍA T. MONTALVO V ${ }^{2}$, \\ ANDREA JIMÉNEZ A ${ }^{1}$, CARLOS ECHIBURÚ-CHAU ${ }^{3}$ and GLORIA M. CALAF ${ }^{3,4}$ \\ ${ }^{1}$ Hospital Gustavo Fricke, Universidad de Valparaíso, Valparaíso; ${ }^{2}$ Facultad de Ciencias \\ de la Salud, ${ }^{3}$ Instituto de Alta Investigación, Universidad de Tarapacá, Arica, Chile; \\ ${ }^{4}$ Center for Radiological Research, Columbia University Medical Center, New York, NY, USA
}

Received April 8, 2008; Accepted June 9, 2008

DOI: 10.3892/or_00000038

\begin{abstract}
Breast cancer is the leading cause of death affecting women worldwide, according to mortality estimation and incidence. In Chile, breast cancer ranks third among cancer mortality rates. Two-hundred and eighty-three breast cancer patients registered at the Gustavo Fricke Hospital of Viña del Mar, Chile, were studied to assess the influence of several factors on the recurrence and survival of breast cancer patients. Patients selected had 5-year post-surgery recurrences of breast cancer and had an average of 58.5 years of age. The variables considered in these patients were the quadrants involved, stage of the tumor, type of recurrence, type of exams, type of surgery, the grade of tumor in relation to Broder's classification and pathology of tumor. The results indicated that the superior external right and left quadrants, Stage IIA, loco-regional recurrences, lumpectomies with axillar lymph node removal and after 5 years, Grade II were prevalent in this population. Among the pathologies analyzed, the ductal carcinomas were the most commonly found and were positive for PCNA, B-catenin, cytokeratin 18 and ErbB2 protein expression. A median follow-up of 60 months of survival was achieved in $95 \%$ of patients. However, those cases with a recurrence of breast cancer had only $40 \%$ survival. The risk of mortality was significantly greater when recurrence was present $(\mathrm{P}<0.0001)$. The global probability of survival of the patients reached $72 \%$ after 5 years. It can be concluded that early detection of breast cancer allows for control of the disease and avoids remissions, or at least extends survival.
\end{abstract}

\section{Introduction}

Breast cancer is the leading cause of death that affects women worldwide (1). In Chile, breast cancer ranks third in cancer

Correspondence to: Dr Gloria M. Calaf, Instituto de Alta Investigación, Universidad de Tarapacá, Calle Antofagasta \#1520, Arica, Chile

E-mail: gmc24@columbia.edu

Key words: recurrence, survival, breast cancer mortality statistics and was found to have increased in 2002 $(2,3)$. Surgery combined with radiotherapy has been the typical treatment for the early stages of breast cancer in order to control loco-regional disease, and $\sim 30 \%$ of the patients have disseminated disease (4-6). To avoid recurrences from micrometastasis, hormone- or chemotherapy adjuvant treatments are often prescribed.

Variables considered of good prognostic value for mortality risk and recurrence of the disease include TNM stage of the disease (7-9), size of tumors (up to $2 \mathrm{~cm}$ in diameter) $\left(\mathrm{T}_{1}\right)$, no lymphatic involvement $\left(\mathrm{N}_{0}\right)$ and absence of metastasis $\left(\mathrm{M}_{0}\right)$. Even in the absence of lymphatic involvement tumors have been found to have $20-30 \%$ of local recurrence when treated with surgery alone $(10,11)$. Radiotherapy has been demonstrated to reduce local recurrence of breast cancer in $70 \%$ of patients after partial mastectomy (11). Advances in the treatment of mammary carcinoma have improved the survival rate of breast cancer patients. Since it is important to know the patterns of recurrence and survival of a population of breast cancer patients, we conducted a retrospective analysis of breast cancer patients registered at the Gustavo Fricke Hospital of Viña del Mar, Chile.

\section{Materials and methods}

Patients and specimens. To evaluate the risk of recurrence after post-surgery of breast cancer, a retrospective analysis was performed on patients registered at the Gustavo Fricke Hospital of Viña del Mar, Chile. Clinical data were obtained from 283 patients who had breast cancer surgery. Two groups were selected from January 1, 1996 to December 31, 2000. One group (19 cases) had no breast cancer recurrence while the other (19 cases) had recurrence after 5 years of follow-up post-surgery. The average age was 58.5 years (range 35-85). The variables identified patients according to the risk of mortality and recurrence of the disease. The patient survival was determined according to the number of persons alive after 5 years of diagnosis. The data parameters analyzed included age, quadrant involved, TNM histological type, type of surgery, nuclear grade histological features according to the Elston and Bloom classification (12) and the presence and time of appearance of loco-regional and distant recurrence. The frequency and distribution of the data used to calculate patient survival were obtained by the log-rank test. 
Regression curves were performed with the Kaplan-Meier method and EpiInfo 6 and statistical product and service solutions (SPSS v.11.0) programs were used.

Immunofluorescent studies. Tissues were prepared as previously reported $(13,14)$ and incubated with anti-mouse antibodies for PCNA (PC10, sc56), ß-catenin (E5, sc6973), cytokeratin 18 (DC10, sc6259) and ErbB2 (RTJ2, sc415) (all Biotechnology Inc., Santa Cruz, CA) at a dilution of 1:500 at $4^{\circ} \mathrm{C}$ overnight. The controls included tissues stained with either the primary or secondary antibodies alone to monitor the background staining. The tissues were viewed on a Zeiss Axiovert 100 TV microscope (Carl Zeiss, Thornwood, NY) using a $40 \mathrm{X} 11.3 \mathrm{NA}$ objective lens equipped with a laser scanning confocal attachment (LSM 410 Carl Zeiss). To excite the rhodamine, an argon/krypton mixed gas laser (488 nm) was used.

\section{Results}

The patterns of recurrence and survival of a population of breast cancer patients were analyzed in a retrospective analysis at the Gustavo Fricke Hospital located in Viña del Mar, Chile. In these studies, the percentage of quadrants most affected, stages of the disease, type of recurrence and type of exams performed in the patients (Table I) were analyzed. The quadrants usually affected by cancer were the superior external right (SER) (28\%) and left (SEL) (27\%) in comparison with the inferior external right (IER) (5\%), superior internal right (SIR) $(7 \%)$, inferior internal right (IIR) $(2 \%)$, inferior external left (IEL) (2\%), superior internal left (SIL) (5\%) and inferior internal left (IIL) (7\%). The most frequent stage of the disease was IIA (35\%) in comparison with Stage 0 (2\%), I (27\%), IIB $(27 \%)$ and III (9\%). Patients had a predominantly locoregional type of recurrence $(68 \%)$ followed by metastasis $(32 \%)$. Clinical diagnosis was predominantly performed $(61 \%)$ while others had image type of exams (39\%). Among the patients considered in this study, 68\% had loco-regional recurrence and $32 \%$ had distant metastasis. Lumpectomy with axillar node removal followed by the Madden procedure was the predominant surgery used in these patients. The nuclear grade that predominated was Grade II of Broder's classification (63.2) and $93.8 \%$ of the cases had lower grades. There was no significant difference in the nuclear grade (data not shown). The ductal carcinomas were the most frequent pathological type found in these studies and most of them were positive for markers such as PCNA, ß-catenin, cytokeratin 18 and ErbB2 protein expression. Fig. 1 shows representative images of markers used in this study. There was no significant difference in relation to the development of recurrence in those patients with estrogen receptor positive (data not shown) breast cancer.

Fig. 2A shows a function of recurrence in relation to time. Results indicated that the probability of recurrence diminished progressively up to 5 years. Fig. 2B shows the survival in patients according to the presence or absence of recurrence after 5 years of follow-up. Cases of breast cancer without any recurrence had $95 \%$ survival after 5 years. However, cases with a recurrence of breast cancer had $40 \%$ survival and indicated that the rise of mortality was significantly greater
Table I. Percentage of quadrants, stages, types of recurrences and exams analyzed in breast cancer patients.

A, Percentage of quadrants

\begin{tabular}{llc}
\hline Abbreviation & \multicolumn{1}{c}{ Quadrant } & Percentage (\%) \\
\hline SER & Superior external right & 28 \\
SEL & Superior external left & 27 \\
SIR & Superior internal right & 7 \\
SIL & Superior internal left & 5 \\
IER & Inferior external right & 5 \\
IEL & Inferior external left & 2 \\
IIR & Inferior internal right & 2 \\
IIL & Inferior internal left & 7 \\
SIER & Superior internal and & 11 \\
& external combined right & \\
SIEL & Superior internal and & 2 \\
& external combined left & \\
Retroareolar & $\quad----------$ & 2 \\
Total & $\quad-----------$ & \\
\hline
\end{tabular}

B, Percentage of stages

\begin{tabular}{lc}
\hline Stage & Percentage (\%) \\
\hline 0 & 2 \\
I & 27 \\
IIA & 35 \\
IIB & 27 \\
III & 9 \\
\hline
\end{tabular}

\section{C, Percentage of types of recurrences}

Type of

recurrence

Percentage $(\%)$

$\begin{array}{ll}\text { Local } & 68 \\ \text { Distant } & 32\end{array}$

D, Percentage of exams used

Exams (type)

Percentage (\%)

Clinical 61

Imaging 39

with recurrence $(\mathrm{P}<0.0001)$. The global probability of survival accumulated for all groups analyzed was $72 \%$ after 5 years of follow-up as is shown in Fig. 2C.

\section{Discussion}

The presence of breast cancer as well as recurrence thereof appear to be multi-factorial in origin. These studies analyzed 


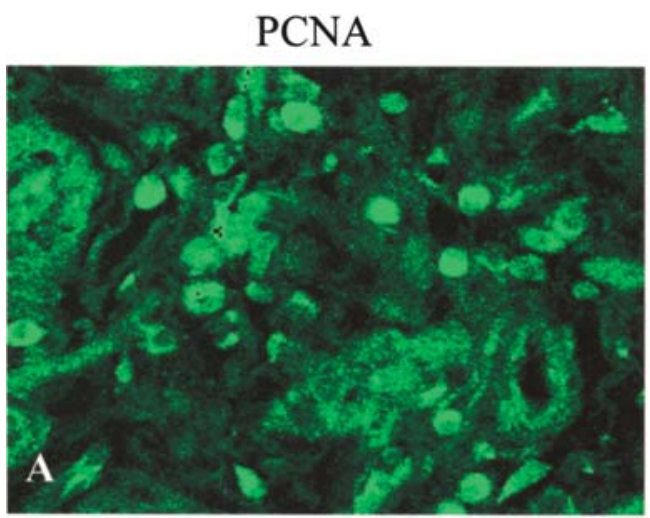

\section{Cytokeratin 18}

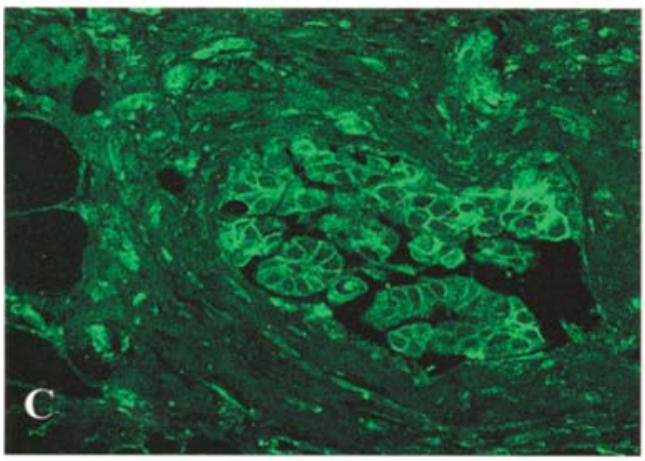

\section{Beta catenin}

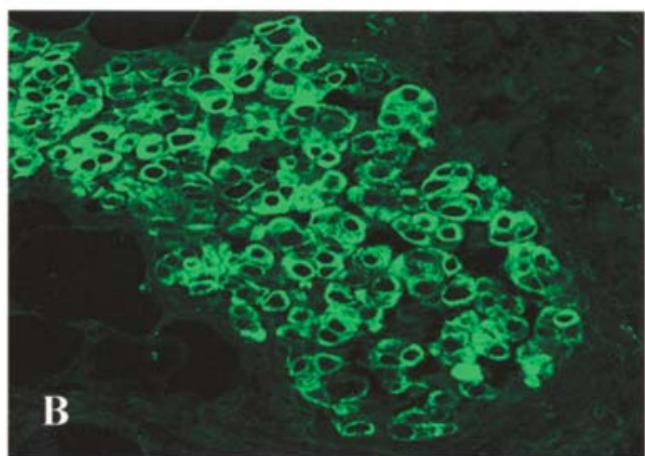

ErbB2

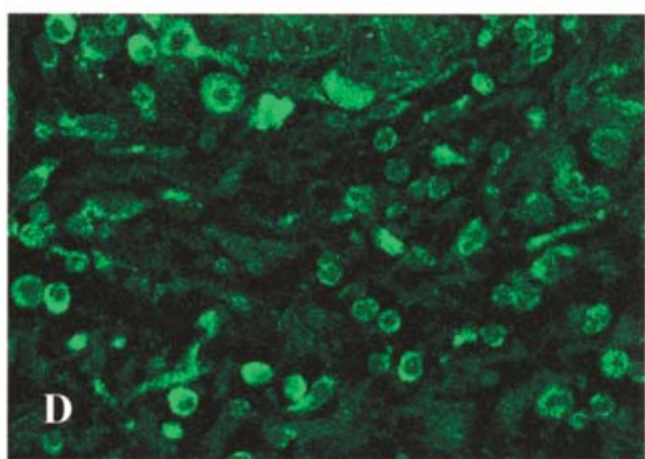

Figure 1. Immunofluorescent images showing the protein expression of (A) PCNA, (B) cytokeratin 18, (C) B-catenin and (D) ErbB2 in infiltrating ductal carcinomas.

\section{Recurrence vs months}

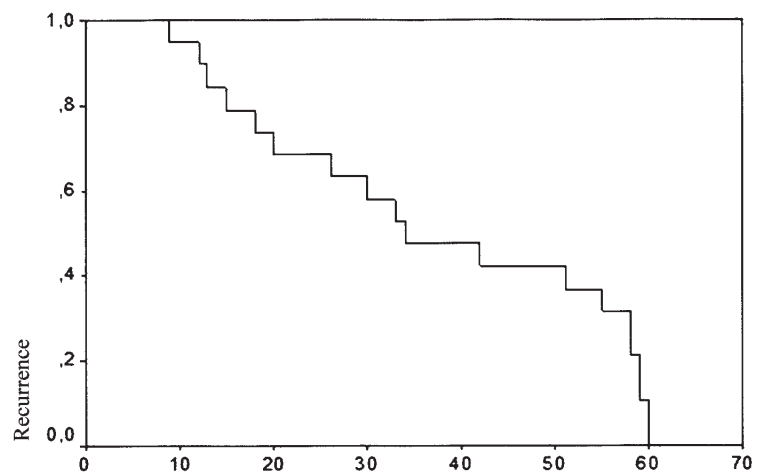

A Months

\section{Survival vs months}

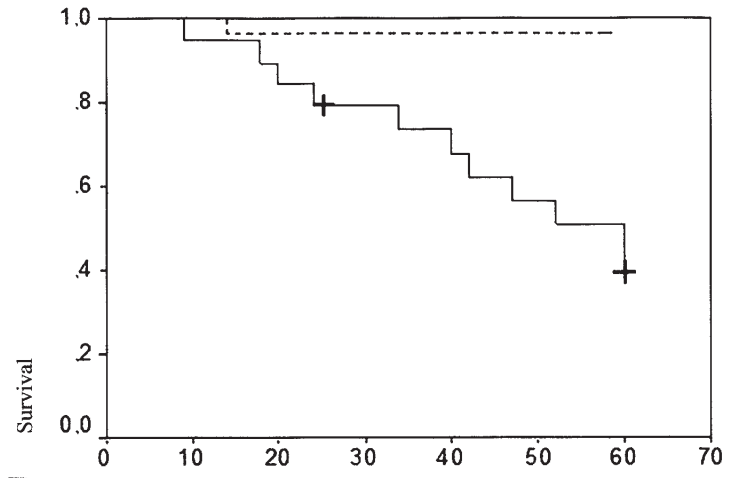

B Months

Global survival

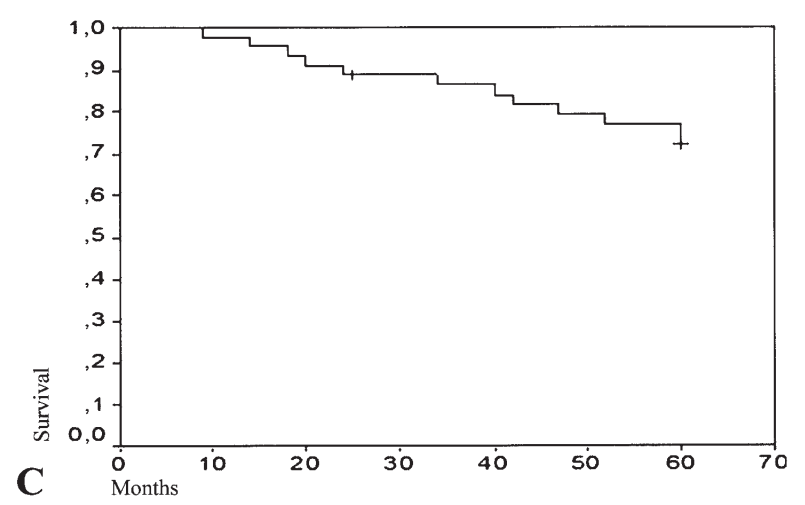

Figure 2. Curves indicating (A) recurrence versus time, (B) survival versus time with and without recurrence and (C) the global survival of 283 patients with breast cancer after 5 -year post-surgery. 
several factors such as the percentage of quadrants, stages, types of recurrence, exams, surgery, nuclear grade, pathological type, presence of estrogen and/or progesterone receptors as well as the function of recurrence in relation to time. The present studies showed that the most common quadrants involved were the superior external either right or left in comparison with the superior internal, inferior external and internal. Studies (15) have considered the location of the tumor as a prognostic factor and it has been found in the superior external quadrants and in the external ones when compared with the internal quadrants or central regions $(16,17)$. It has been suggested that there is a tendency for patients to develop tumors in places directly proportional to the amount of mammary gland tissue found in each quadrant (15). Molecular studies have shown that there is genomic instability in external quadrants of the breast suggesting a tendency in patients to develop breast cancer in such areas (15).

Other authors (18) have considered tumor stages as a good prognostic tool of recurrence and risk of death. The increment in tumor stage implied an increase of 2.8 times the risk of mortality (18). Among the patients considered in this study, 68\% had loco-regional recurrence. It has been reported that there were no significant differences between local or distant recurrences in patients with central or retro-areolar tumors treated with mastectomy or lumpectomy $(19,20)$.

The tendency to minimize surgical procedures performed in breast cancer, classified as $\mathrm{T}_{1} \mathrm{~N}_{0} \mathrm{M}_{0}$, have necessitated researchers to increase their efforts to identify pathological factors of high risk in primary lesions (21). Lumpectomy with axillar removal was the most common surgery used in patients in the present study. Several studies (22-24) have shown that multiple factors influence the risk of recurrence of breast cancer patients subjected to conservative surgery. Thus, given the presence of local or distant recurrence in patients with this type of disease, mortality has been increasing during the last 20 years (25).

Nuclear grade II of Broder's classification was the most common grade found in the group studied (63\%). In the present study no relationship between the nuclear grade and recurrence was found. However, other studies (21) have reported on a correlation between survival and nuclear grade in the primary lesion. Other authors (26) have shown that the recurrence of the disease increased progressively with a high nuclear grade (21).

It is known that early detection of breast cancer is the basis of follow-up programs that use image analysis and biological markers (27). These data suggest that early detection of breast cancer allows for control of the disease and avoids remissions, or at least extends survival (28). Certain reports have considered the patterns of expression of receptors in identifying aggressive treatments $(29,30)$. Authors have indicated that the pattern of ER-positive/PR-negative/HERpositive expression is associated with a great number of recurrences in the early stage of the disease (31). The ER status has been related to local recurrence in studies of patients treated with conservative breast surgery and adjuvant therapy (32-34). Studies have indicated that tamoxifen and the ER status are independently associated with local recurrence $(35,36)$.
The probability of no recurrence diminished progressively until the 5-year analysis. Furthermore, it was found that those cases of cancer with no recurrence had $95 \%$ survival, but only $40 \%$ in those cases with recurrence after a 5-year follow-up. The global probability of survival reached $72 \%$. Recurrence of breast cancer was associated with an increase in mortality in women. Other retrospective studies have indicated that the mortality rate was greater in women with distant metastasis in 10 years of survival than those with local recurrence (37). According to many reports, the 5-year survival rate has increased in breast cancer patients in the last two decades (38). In general, a decrease in the mortality due to early detection (39) and to the increment of hormonal and adjuvant therapies has been demonstrated (40).

Ductal carcinomas were the most common histological type found in the group studied. It was noteworthy to find that this type of carcinoma was positive for the proliferative index PCNA, cytokeratin 18 and ErbB2 as we have previously shown (13) in cell lines such as MCF-10F, where a spontaneously immortalized human breast epithelial cell line was transformed with benzo(a)pyrene and was transfected with c-Ha-ras oncogene. In such studies, the protein expression was also determined by immunofluorescent staining coupled with confocal microscopy. Previous results have also shown an increased $B$-catenin protein expression in the same cell lines (14). The expression of $B$-catenin was significantly associated with c-erbB2 overexpression (41). Loss of junctional $ß$-catenin has often been observed in breast cancer and correlated with poor outcome.Also in our study ß-catenin expression was associated with c-erbB2 overexpression. It has been reported that $\beta$-catenin may be phosphorylated directly by ErbB2 and translocated to the cytoplasm and nucleus (41). Changes in $\beta$-catenin phosphorylation may compromise adhesion in cadherin-positive cancers and facilitate the invasion and metastasis of many cancer cells.

\section{Acknowledgements}

The authors would like to thank Danissa Barahona for her secretarial assistance. We are sincerely thankful for support provided by Fondecyt grant \#1080482.

\section{References}

1. Instituto Nacional de Estadísticas (INE). Ministerio de Salud. Registro Civil, 2001.

2. Lee K, Schwartz R, Iglesis R, Vélez R and Gómez L: Cáncer de mama oculto: dos casos clínicos analizados según el concepto actual. Rev Med Chile 134: 1166-1170, 2006.

3. Orellana C, Torres S, Derio L and Prieto M: Cancer care in Chile. Lancet Oncol 4: 653-656, 2003.

4. Cady B, Stone MD, Schuler JG, Thakur R, Wanner MA and Lavin PT: The new era in breast cancer: invasion, size, and nodal involvement dramatically decreasing as a result of mammographic screening. Arch Surg 131: 301-308, 1996.

5. Fisher B: Cancer of the breast: size of neoplasm and prognosis. Cancer 24: 1071-1080, 1969.

6. Joensuu $\mathrm{H}$ and Toikkanen S: Comparison of breast carcinomas diagnosed in the 1980's with those diagnosed in the 1940's to 1960's. Br Med J 303: 155-158, 1991.

7. Yarbro J, Page D and Fielding L: American joint committee on cancer prognostic factors consensus conference. Cancer 86: 2436-2446, 1999.

8. Singletary E, Allred C, Ashley P, et al: Revision of the American Joint Committee on cancer staging system for breast cancer. J Clin Oncol 20: 3628-3636, 2002. 
9. Sanchez C, Bustos M, Camus M, et al: ¿Es curable el cáncer de mama en etapa precoz?. Resultados del tratamiento combinado con cirugía, radioterapia y quimioterapia. Rev Med Chile 135: 427-435, 2007.

10. Valagussa P, Bonadonna G and Veronesi U: Patterns of relapse and survival following radical mastectomy. Analysis of 716 consecutive patients. Cancer 41: 1170-1178, 1978.

11. Vinh-Hung V and Verschraegen C: Breast-conserving surgery with or without radiotherapy: Pooled-analysis for risks of ipsilateral breast tumor recurrence and mortality. J Natl Cancer Inst 96: 115-1121, 2004.

12. Bloom $\mathrm{H}$ and Richardson W: Histological grading and prognosis in breast cancer. A study of 1409 cases of which 359 have been followed for 15 years. Br J Cancer 2: 359-377, 1957.

13. Calaf GM, Alvarado ME and Hei TK: Oncoprotein expression and morphological phenotypes of human breast epithelial cells transformed by the c-Ha-ras oncogene. Oncol Rep 14: 885-893, 2005.

14. Calaf GM, Alvarado ME and Hei TK: Beta catenin is associated with breast cancer progression in vitro. Int J Oncol 26: 913-921, 2005.

15. Ellsworth D, Ellsworth R, Love B, et al: Outer breast quadrants demonstrate increased levels of genomic instability. Ann Surg Oncol 11: 861-868, 2004.

16. Bevilacqua J, Cody Hsi, Macdonald K, Tan L, Borgen P and Van Zee K: A model for predicting axillary node metastases based on 2000 sentinel node procedures and tumour position. Eur J Surg Oncol 28: 490-500, 2002.

17. Janni W, Rack B, Sommer H, et al: Intra-mammary tumor location does not influence prognosis but influences the prevalence of axillary lymph-node metastases. J Cancer Res Clin Oncol 129: 503-510, 2003.

18. Saxe G, Rock C, Wicha M and Schottenfeld D: Diet and risk for breast cancer recurrence and survival. Breast Cancer Res and Treat 53: 241-253, 1999.

19. Donnelly J, Mack P and Donaldson L: Follow-up of breast cancer: time for a new approach? Int J Clin Pract 55: 431-433, 2001.

20. Hiramanek N: Breast cancer recurrence: follow-up after treatment for primary breast cancer. Postgrad Med J 80: 172-176, 2004.

21. Bilik R, Mar C, Wolloch Y and Dintsman M: Histopathologic high risk factors influencing the prognosis of patients with early breast cancer (T1NOM0). Am J Surg 151: 460-464, 1986.

22. Grunfeld E, Gray A and Mant D: Follow-up of breast cancer in primary care vs. specialist care: results of an economic evaluation. Br J Cancer 79: 1227-1233, 1999.

23. Hughes L and Courtney S: Follow-up of patients with breast cancer. Br Med J 290: 1229-1230, 1985.

24. Loprinzi C: Follow-up testing for curatively treated cancer survivors. What to do? JAMA 273: 1877-1878, 1995.

25. Sakorafas G, Tsiotou A and Pavlakis G: Follow-up after primary treatment for breast cancer. Acta Oncol 39: 935-940, 2000.

26. Fracchia A, Rosen P and Ashikari R: Primary carcinoma of the breast without axillary lymph node metastases. Surg Gynecol Obstet 151: 375-378, 1980.
27. The Givio Investigators: Impact of follow-up testing on survival and health-related quality of life in breast cancer patients. JAMA 271: 1587-1592, 1994.

28. Schapira D: Breast cancer surveillance-a cost-effective strategy. Breast Cancer Res Treat 25: 107-111, 1993.

29. Provenzano E, Hopper J, Giles G, Marr G, Venter DJ and Armes JE: Biological markers that predict clinical recurrence in ductal carcinoma in situ of the breast. Eur J Cancer 39: 622-630: 2003.

30. Lebeau A, Unholzer A, Amann G, et al: EGFR, HER-2/neu, cyclin D1, p21 and p53 in correlation to cell proliferation and steroid hormone receptor status in ductal carcinoma in situ of the breast. Breast Cancer Res Treat 79: 187-198, 2003.

31. Kepple J, Henry-Tillman RS, Klimberg VS, Layeeque R, Siegel E, Westbrook K and Korourian S: The receptor expression pattern in ductal carcinoma in situ predicts recurrence. Am J Surg 192: 68-71, 2006.

32. Swenson KK, Decher L, Haselow R, Farrell JB and Sperduto PW: Prognostic factors after conservative surgery and radiation therapy for early stage breast cancer. Am J Clin Oncol 21: 111-116, 1998.

33. Fowble B, Fein DA, Hanlon AL, et al: The impact of tamoxifen on breast recurrence, cosmesis, complications, and survival in estrogen receptor-positive early-stage breast cancer. Int J Radiat Oncol Biol Phys 35: 669-677, 1996.

34. Mansfield C, Komarnicky L, Schwartz G, et al: Ten-year results in 1070 patients with stages I and II breast cancer treated by conservative surgery and radiation therapy. Cancer 75: 2328-2336, 1995.

35. Yaghan R, Stanton PD, Robertson KW, Going JJ, Murray GD and McArdle CS: Oestrogen receptor status predicts local recurrence following breast conservation surgery for early breast cancer. Eur J Surg Oncol 24: 424-426, 1998.

36. Tartter P, Kaplan J, Bleiweiss I, Gajdos C and Kong A: Lumpectomy margins, reexcision, and local recurrence of breast cancer. Am J Surg 179: 81-85, 2000

37. Carlson G, Page A, Johnson E, Nicholson K, Styblo T and Wood W: Local recurrence of ductal carcinoma in situ after skinsparing mastectomy. J Am Coll Surg 204: 1074-1080, 2007.

38. Sarker M, Jatoi I and Becher H: Racial differences in breast cancer survival in women under age 60 . Breast Cancer Res Treat 106: 135-141, 2007.

39. Weir HK, Thun MJ, Hankey BF, et al: Annual report to the nation on the status of cancer, 1975-2000, featuring the uses of surveillance data for cancer prevention and control. J Natl Cancer Inst 95:1276-1299, 2003.

40. Mariotto A, Feuer E, Harlan L, Wun L, Johnson K and Abrams J: Trends in use of adjuvant multi-agent chemotherapy and tamoxifen for breast cancer in the United States: 1975-1999. J Natl Cancer Inst 94: 1626-1634, 2002.

41. Zhang Y-G, Du J, Tian X-X, Zhong Y-F and Fang W-G: Expression of E-cadherin, beta-catenin, cathepsin D, gelatinases and their inhibitors in invasive ductal breast carcinomas. Chin Med J 120: 1597-1605, 2007. 